\title{
Useful websites
}

Annual Business Inquiry

Annual Population Survey

Annual Survey of Hours and Earnings

Business Enterprise Research and
Development Expenditure

Communities and Local Government

Department for Children, Schools and Families

Department for Culture, Media and Sport

Department of Energy and Climate Change

Department for Environment, Food and Rural Affairs

Department for Social Development in Northern Ireland

Department for Transport

Department for Work and Pensions

Department of Enterprise, Trade and

Investment, Northern Ireland

Department of Education, Northern Ireland

Department of Health

Department of the Environment, Northern Ireland

Designated Areas:

England

Wales

Scotland

Northern Ireland

Economic and Labour Market review

England and Wales Census

Environment Agency

General Household Survey

General LiFestyle Survey

General Register Office for Scotland

Geography in National Statistics

Home Office

Index of Multiple Deprivation www.statistics.gov.uk/abi

www.statistics.gov.uk/statbase/Product.asp?vlnk=10855

www.statistics.gov.uk/statbase/product.asp?vlnk=13101

www.statistics.gov.uk/statbase/Product.asp?vlnk=8206

wWw.communities.gov.uk

www.dcsf.gov.uk

www.culture.gov.uk

www.decc.gov.uk/en/content/cms/statistics/statistics.aspx

www.defra.gov.uk

www.dsdni.gov.uk/

www.dft.gov.uk/pgr/statistics

www.dwp.gov.uk

www.detini.gov.uk

www.deni.gov.uk/

www.dh.gov.uk

www.doeni.gov.uk/

www.defra.gov.uk/evidence/statistics/index.htm

www.ccw.gov.uk/

www.snh.org.uk/

www.doeni.gov.uk/

www.statistics.gov.uk/elmr

www.statistics.gov.uk/census/

wWw.environment-agency.gov.uk

www.statistics.gov.uk/StatBase/Product.asp?vlnk=5756

www.statistics.gov.uk/StatBase/Product.asp?vlnk=5756

www.gro-scotland.gov.uk

www.statistics.gov.uk/geography/default.asp

www.homeoffice.gov.uk

www.communities.gov.uk/indices 
Labour Force Survey

Land Registry

Learning and Skills Council

Life Expectancy estimates

Migration statistics

Ministry of Justice

Neighbourhood Statistics:

England and Wales

Scotland

Northern Ireland

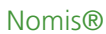

Northern Ireland Statistics and Research Agency

Office for National Statistics

Population Estimates by Ethnic Group

Population Estimates

Publication Hub

Home Page

Release Calendar

Regional Statistics

What are National Statistics?

UK Statistics Authority

Regional Economic Indicators

Regional Trends

Regional Trends - Online Tables (latest data)

Scottish Environment Protection Agency

Scottish Government

Scottish Statistics

Statistics for Regions in England:

North East

North West

Yorkshire and The Humber

East Midlands

West Midlands

East of England

London

South East

South West

Subnational Population Estimates for England

The NHS Information Centre for health and social care

Welsh Assembly Government www.ons.gov.uk/about/who-we-are/our-services/unpublished-data/social-survey-data/lfs/lfs-faqs

www.landregistry.gov.uk

Www.Isc.gov.uk Life

www.statistics.gov.uk/statbase/Product.asp?vlnk=8841

www.statistics.gov.uk/statbase/Product.asp?vlnk=15108

www.justice.gov.uk

www.neighbourhood.statistics.gov.uk/

WWW.sns.gov.uk/

www.ninis.nisra.gov.uk/

www.nomisweb.co.uk/

www.nisra.gov.uk/

www.ons.gov.uk

www.statistics.gov.uk/StatBase/Product.asp?vlnk=14238

www.statistics.gov.uk/statbase/Product.asp? $v$ Ink=601\&More $=\mathrm{N}$

www.statistics.gov.uk/hub/index.html

www.statistics.gov.uk/hub/release-calendar/index.html

www.statistics.gov.uk/hub/regional-statistics/index.html

www.statistics.gov.uk/hub/what-are-national-statistics-/index.html

wWw.statisticsauthority.gov.uk/

www.statistics.gov.uk/StatBase/Product.asp?vlnk=9472

www.statistics.gov.uk/regionaltrends

wWw.statistics.gov.uk/regionaltrends/data

www.sepa.org.uk

www.scotland.gov.uk

www.scotland.gov.uk/Topics/Statistics

www.statistics.gov.uk/cci/nugget.asp? $\mathrm{id}=1072$

www.statistics.gov.uk/cci/nugget. asp? $\mathrm{id}=1073$

www.statistics.gov.uk/cci/nugget.asp? $\mathrm{id}=1074$

www.statistics.gov.uk/cci/nugget.asp? $\mathrm{id}=1075$

www.statistics.gov.uk/cci/nugget.asp? $\mathrm{id}=1076$

www.statistics.gov.uk/cci/nugget.asp? $\mathrm{id}=1077$

www.statistics.gov.uk/cci/nugget.asp? $\mathrm{id}=1078$

wWw.statistics.gov.uk/cci/nugget.asp? $i d=1079$

wWw.statistics.gov.uk/cci/nugget.asp? $\mathrm{id}=1080$

www.statistics.gov.uk/statbase/Product.asp?vlnk=997

www.ic.nhs.uk

www.wales.gov.uk 